\title{
Surface and structural similarity in analogical transfer
}

\author{
KEITH J. HOLYOAK \\ University of Califormia, Los Angeles, California \\ and \\ KYUNGHEE KOH \\ University of Michigan, Ann Arbor, Michigan
}

\begin{abstract}
Two experiments investigated factors that influence the retrieval and use of analogies in problem solving. Experiment 1 demonstrated substantial spontaneous analogical transfer with a delay of several days between presentation of the source and target analogues. Experiment 2 examined the influence of different types of similarity between the analogues. A mechanism for retrieval of source analogues is proposed, based on summation of activation from features shared with a target problem. The results of Experiment 2 indicated that both structural features, which play a causal role in determining possible problem solutions, and salient surface features, which do not have a causal role, influence spontaneous selection of an analogue. Structural features, however, have a greater impact than do surface features on a problem solver's ability to use an analogue once its relevance has been pointed out.
\end{abstract}

A person confronted by a novel problem can sometimes solve it by drawing an analogy to a similar problem that has a known solution. Analogy is a central form of induction used to generate inferences in pragmatically important situations (Holland, Holyoak, Nisbett, \& Thagard, 1986). Analogical transfer is one factor that makes human problem solvers more flexible than current expert systems in artificial intelligence. Such systems are "brittle" in that they typically require extensive intervention by the human programmer in order to deal with relatively small changes in problem domains. An understanding of the mechanisms of human analogical transfer, in addition to providing insight into human cognition, might suggest remedies for the brittleness of mechanized problem solvers.

At a global level, analogy is used to generate knowledge applicable to a novel target domain by transferring knowledge from a source domain that is better understood. Analogical problem solving can be characterized in terms of four basic steps: (1) constructing mental representations of the source and the target; (2) selecting the source as a potentially relevant analogue to the target; (3) map-

This research was supported by National Science Foundation Grant BNS-8216068 and Army Research Institute Contract MDA903-86-K0297. The first author held an NIMH Research Scientist Development Award, 1-K02-MH00342-05. Early drafts of the paper were written while the first author was visiting the Psychology Department at CarnegieMellon University and the Learning Research and Development Center of the University of Pittsburgh. Patricia Cheng and Edward Smith provided useful comments on earlier drafts of the paper. A talk based on the paper was presented at the annual meeting of the Midwestern Psychological Association, May 1986, in Chicago. Reprint requests may be sent to K. J. Holyoak, Department of Psychology, University of California at Los Angeles, Los Angeles, CA 90024. ping the components of the source and target; and (4) extending the mapping to generate a solution to the target (Holyoak, 1984). These steps need not be carried out in a strictly serial order, and they may interact in many ways (e.g., the selection step may require some preliminary mapping); nonetheless, they provide a useful conceptual organization for the overall process.

Perhaps the least understood of the above four steps is the second, selecting a source analogue. Selection requires retrieval of the source analogue from memory and noticing of its relevance to the target problem. Particularly in the case of analogies between problems drawn from disparate domains, it is unclear how a problem solver can retrieve a potentially useful source analogue from a large knowledge base. Computational models of analogy have typically evaded this issue, either by explicitly directing the program to compare particular situations (Burstein, 1986) or by implementing a psychologically implausible exhaustive search mechanism (Winston, 1980). Carbonell (1983) suggested that retrieval of problem analogies could be facilitated by organizing the data base according to similarities in basic problem components, such as goals, starting states, and problem constraints; however, the usefulness of such a scheme has not been demonstrated by either computational or psychological evidence.

\section{Evidence for Spontaneous Use of Analogies}

There appear to be a variety of mechanisms by which people can potentially select plausible source analogues. In some cases source analogues are generated by systematic transformations of the target (Clement, 1982). In other cases the source will be directly provided by a teacher, as is the case, for example, when water flow is used as 
an analogy to elucidate the nature of electricity (Gentner \& Gentner, 1983). In the present paper we will focus on the processes by which an analogist, without the intervention of a teacher, may retrieve the representation of a situation stored in memory and notice its relevance to a target problem.

The conditions under which people are likely spontaneously to make use of potential analogies are far from clear. Indeed, a consistent research finding has been that college subjects often fail to use analogies spontaneously (Gick \& Holyoak, 1980, 1983; Hayes \& Simon, 1977; Reed, Ernst, \& Banerji, 1974). For example, Gick and Holyoak 1980, 1983) had subjects attempt to solve Duncker's (1945) radiation problem, which involves finding a way for a doctor to use $\mathrm{X}$ rays to destroy a stomach tumor without damaging the surrounding healthy tissue. Some groups of subjects first read a story describing an analogous military problem in which a general captured a centrally located fortress by having small units of soldiers attack simultaneously along multiple roads. When given a hint to use the prior military story, about $75 \%$ of the subjects in a typical experiment were able to generate the analogous convergence solution to the radiation problem (i.e., focusing multiple low-intensity $\mathrm{X}$ rays on the tumor from different directions). In contrast, only about $30 \%$ of subjects generated this solution prior to receiving an explicit hint. Given that about $10 \%$ of subjects produced the convergence solution without any analogue, this suggests that only about $20 \%$ of the subjects may have spontaneously retrieved and applied the analogy.

In fact, one could reasonably question whether there is any convincing experimental evidence that people notice analogies between problems presented in substantially remote contexts. Even in the case of analogies between problems in the same domain, such as geometry, anecdotal reports suggest that students seldom notice analogies between problems presented in different chapters of their textbook. In virtually all the experiments reported to date, the source and target analogues were presented consecutively within a single experimental session. It could be, for example, that the $20 \%$ of subjects in Gick and Holyoak's $(1980,1983)$ experiments who spontaneously used the analogy did so simply because they were sensitive to demand characteristics of the situation, which would surely suggest that the story and the problem immediately following might be somehow related. Spencer and Weisberg (1986) found no evidence of transfer to the radiation problem when a delay or change of context separated presentation of the source and target problems.

Perhaps the strongest evidence that analogues drawn from remote domains sometimes produce spontaneous transfer comes from studies in which multiple source analogues are provided. Gick and Holyoak (1983) had some groups of subjects first read two convergence stories (e.g., the military story described above and a fire-fighting story in which converging sources of fire retardant were used to extinguish a large blaze). Other groups read a single convergence story plus a nonanalogous story. All sub- jects summarized each story and also wrote descriptions of how the two stories were similar. The latter task was intended to trigger a mapping between the two stories, which would have the incidental effect of leading to the induction of an explicit representation of the shared schematic structure. All subjects then attempted to solve the X-ray problem, both before and after a hint to consider the stories. Gick and Holyoak (1983) found that subjects in the two-analogue groups were significantly more likely to produce the convergence solution, both before and after the hint, than were subjects in the one-analogue groups. Since demand characteristics were presumably comparable for both sets of subjects, the advantage of the two-analogue subjects prior to the hint is evidence of spontaneous transfer.

Gick and Holyoak (1983) interpreted these and other more detailed results to indicate that induction of an explicit schema facilitates transfer. Once a person has induced a schema from initial examples, novel problems that can be categorized as instances of the schema can be solved without necessarily directly accessing representations of the initial analogues. It follows that although experiments illustrating the role of schemata demonstrate spontaneous interdomain transfer, they do not provide clear evidence of analogical transfer, in the sense of direct transfer from a representation of a particular prior situation to a novel problem. A major goal of the present study was to identify conditions under which spontaneous analogical transfer in fact occurs.

\section{Surface and Structural Similarity}

If two situations drawn from disparate domains have never previously been associated, there can be no direct retrieval pathway linking the two. How, then, might the target activate the source? This question pertains not only to analogical problem solving, but more generally to mechanisms by which an episode can trigger reminding of a disparate but structurally similar situation (Schank, 1982).

One possibility is that retrieval of analogies is based on summation of activation resulting from multiple shared features. Summation mechanisms have been proposed in many activation-based cognitive models to account for performance in tasks requiring retrieval or classification of inputs (e.g., Anderson, 1983; McClelland \& Rumelhart, 1981; Thibadeau, Just, \& Carpenter, 1982). The present proposal for extending the summation mechanism to account for retrieval of analogies could be implemented in various ways, and will be described in general terms. We assume that situations can be represented in terms of more elementary features (a term we will use in a general sense to include both properties and relations). Each feature attended to will activate memory representations of other situations that share that feature. Shared features thus serve as retrieval cues in a content-addressable memory system. Activation from multiple shared features will summate, and if the activation level of a stored representation exceeds some threshold, that representa- 
tion will become available for further processing, such as initiation of an explicit mapping process. Such a summation mechanism allows multiple weak cues to collaborate as converging evidence indicating the potential relevance of related situations stored in memory. ${ }^{1}$

It might seem that such a simple summation mechanism would tend to retrieve stored representations with many superficially similar features, rather than remote analogues with important shared structural components. In fact, the low rate of spontaneous transfer observed by Gick and Holyoak $(1980,1983)$ and others suggests that this possibility may be at least partially realized. An experiment by Gilovich (1981) provided evidence of the effect of superficial cues on the retrieval of analogues. He had subjects suggest resolutions for various hypothetical political crises, and found the subjects' suggestions were influenced by superficial resemblances between the hypothetical crises and actual historical situations. Ross (1984) found evidence that superficial similarity influences retrieval of examples in the domain of statistics problems.

In order to investigate the conditions under which people will retrieve a source analogue and notice its relevance to a target problem, in the present study we manipulated different types of similarity, which will be termed surface and structural. Following Hesse (1966) and Tversky (1977), we assume that similarity between two situations can be decomposed into identities and differences between features. The surface versus structural distinction depends on whether or not a feature is causally relevant to goal attainment. A surface dissimilarity between two situations involves what Holyoak (1984) termed a structurepreserving difference-a change in a feature that does not influence goal attainment. For example, in the convergence analogies investigated by Gick and Holyoak (1980), there are many differences between the fortress attacked by the general and the tumor attacked by the doctor; but because the only causally relevant aspect is that each is a centrally located target, these differences are structure preserving.

In contrast, a structural dissimilarity involves a structure-violating difference that alters the causal relations in the two situations. Suppose, for example, that the radiation problem stated that only one $X$-ray source was available. This difference would block use of multiple converging forces, and hence would violate the structure of the solution plan paralleling that which succeeded in the military story. Such a structural dissimilarity would be expected to make the analogy less useful. ${ }^{2}$ Note that the present distinction between surface and structural features is defined in terms of the causal relationships involved in problem situations, rather than in terms of purely syntactic criteria, such as Gentner's (1983) distinction between one-place and multiplace predicates. (See Holyoak, 1985 , for a critique of purely syntactic analyses of analogy.)

Ideally, a problem solver would use only the structural features of the target as retrieval cues, thus avoiding activation of superficially similar but unhelpful situations.
Carbonell's (1983) proposal that situations are indexed by problem components (which constitute structural features) thus has considerable normative appeal. In practice, however, the problem solver's ability to distinguish surface from structural features will almost inevitably be imperfect, since initial understanding of the unfamiliar target problem will be impoverished. Consequently, surface features that in fact are functionally irrelevant to a solution to the target problem may affect the solution plan indirectly by influencing the selection of a source analogue, as suggested by the results of Gilovich (1981) and Ross (1984).

It should now be clear why it is generally difficult for people to spontaneously access relevant source analogues from disparate domains. The basic problem is that a remote analogue, by definition, shares few of the salient surface features of the target. To the extent that these features serve as retrieval cues, they will tend to activate competing associations that may block retrieval of more remote analogues. Conversely, the more the problem solver is able to identify and focus on the causally relevant aspects of the target problem, the greater the probability that a useful but remote analogue will be retrieved.

Once a source analogue has been retrieved, surface features should have less impact on the subsequent mapping process than will structural ones. Structure-violating differences will necessitate refinement of the initial solution plan generated by the mapping, whereas structurepreserving differences will not. Thus, surface features will tend to have a relatively greater impact on selection of a source analogue than on the subsequent mapping process. For example, it seems much easier to learn about electrical circuits by mapping them with water systems than to spontaneously link the two analogues in the first place. In contrast, structure-violating differences should diminish not only the probability of selecting the source analogue, but also the probability of using it successfully once mapping is initiated. The predicted effects of surface and structural similarity were tested in Experiment 2.

\section{EXPERIMENT 1}

Experiment 1 was designed to demonstrate that spontaneous analogical transfer can be obtained in the absence of demand characteristics that might serve as cues to relate the source and target analogues. The basic strategy was to impose a substantial delay between presentation of the source and the target, and to alter the context in which each was presented. Establishing that spontaneous transfer can be reliably obtained is a precondition for investigating the relationship between types of similarity and transfer.

\section{Method}

Materials. A new convergence analogue was written for this purpose. It was first written as a story describing a problem and its solution, and was then modified for use in Experiment 1 by deleting the solution and presenting the problem as a target for subjects 
to solve. The basic content of this "lightbulb story" was inspired by an analogy spontaneously mentioned in a protocol obtained earlier from a subject solving the radiation problem (Gick \& Holyoak, 1980, p. 328). In this story (see Appendix), the filament of an expensive lightbulb in a physics lab was broken. The lightbulb was completely sealed, but an intense laser could be used to fuse the filament. However, at that high intensity, the glass surrounding the filament would be broken. At lower intensities the laser would not break the glass, but neither would it affect the filament. The solution was to direct multiple low-intensity lasers toward the filament from different directions. Table 1 illustrates the analogical correspondences between the lightbulb story and the radiation problem.

Pilot data indicated that subjects who first read the lightbulb story would often spontaneously produce the corresponding convergence solution to the radiation problem. The present experiment was performed to exclude an explanation of such transfer in terms of demand characteristics.

Design and Procedure. Two groups of subjects were tested, all of whom were currently enrolled in an introductory psychology course at the University of Michigan. The course was taught in small sections by different instructors, using various textbooks. Subjects in the analogy condition were selected from sections in which the textbook was Psychology by Gleitman (1981). This text contains an extensive discussion of Duncker's (1945) investigation using the radiation problem, and includes an illustrated explanation of the convergence solution (pp. 321-322). For these subjects, the textbook treatment and class discussion of the radiation problem provided an incidental context in which they learned a potential source analogue. Subjects in the control condition were selected from sections of the course using other textbooks, which did not describe the radiation problem. These subjects thus were not provided with a source analogue. Since students were assigned to sections without regard to the textbook used, there was no reason to expect analogy and control subjects to differ with respect to other factors that might influence performance.

From 3 to 7 days after the subjects in the analogy group had discussed the radiation problem in class, all subjects were brought into the lab in small groups to serve in the experiment. They were told that the experiment involved solving problems. The first problem was a version of the lightbulb story described above with the solution omitted. Subjects read the problem, which described the broken lightbulb in a physics lab, and were asked to suggest procedures by which the laser could be used to fuse the filament. They were asked to write down as many solutions as possible, and to not worry if they were unsure whether a possible solution was actually feasible. No hint was given for them to relate the problem to material in their course. When they had completed the lightbulb problem, they were given the radiation problem, stated as in Gick and Holyoak (1980), and were asked to provide possible solutions. This was done in order to ascertain whether subjects in the analogy condition had in fact learned the convergence solution to the radiation problem from their textbook.
Subjects. Twenty-one subjects served in the analogy condition and 10 served in the control condition. Subjects received course credit for participating in the experiment.

\section{Results and Discussion}

Of subjects in the analogy condition, $81 \%$ produced the convergence solution to the lightbulb problem, and $86 \%$ produced the convergence solution to the radiation problem. The corresponding percentages for subjects in the control group were $10 \%$ and $10 \%$. A solution was scored as indicating convergence as long as the idea of administering lasers (or $\mathrm{X}$ rays) from different directions was clearly stated. Differences in solution frequencies were tested using the maximum-likelihood chi-square statistic. Subjects in the analogy group were much more likely than those in the control group to produce the convergence solution to the lightbulb problem $[81 \%$ vs. $10 \%$; $\left.G^{2}(1)=15.2, p<.001\right]$, indicating substantial spontaneous transfer from the source analogue encountered days earlier in a textbook to the target problem. Furthermore, the analogy subjects were also much more likely than control subjects to produce the convergence solution to the radiation problem itself, confirming that they had in fact learned the solution.

The 4 subjects in the analogy group who failed to generate the convergence solution to the lightbulb problem included the 3 who failed to generate the convergence solution to the radiation problem. Most of the failures to transfer the solution from the source to the target are therefore attributable to subjects who may have failed to encode the source analogue in the first place.

\section{EXPERIMENT 2}

The results of Experiment 1, in contrast to earlier findings, such as those of Gick and Holyoak $(1980,1983)$ and Spencer and Weisberg (1986), revealed a high frequency of spontaneous analogical transfer, even when a delay of several days was imposed between presentation of the source and target analogues. The most probable factor leading to greater transfer in the present study is that the lightbulb problem is in several respects more similar to the radiation problem than were the story analogues used in earlier work. One difference in similarity involves the instruments used in the analogues. A laser is obvi-

Table 1

Correspondences Between Lightbulb Story and Radiation Problem

\section{Lightbulb Story}

Initial State

Goal: Use lasers to fuse filament

Resources: Sufficiently powerful laser

Operators: Reduce laser intensity, move laser source, activate lasers

Constraint: High-intensity laser will break glass

Solution Plan: Administer low-intensity lasers from multiple directions simultaneously

Outcome: Filament fused by lasers

\section{Radiation Problem}

Initial State

Goal: Use X rays to destroy tumor

Resources: Sufficiently powerful rays

Operators: Reduce ray intensity, move ray source, administer rays

Constraint: High-intensity rays will destroy healthy tissue

Solution Plan: Administer low-intensity rays from multiple directions simultaneously

Outcome: Tumor destroyed by $\mathrm{X}$ rays 
ously far more similar to $\mathrm{X}$ rays than an army is, providing a significant additional retrieval cue in the case of the lightbulb story. In addition, the deeper structural parallels between the lightbulb and radiation analogues make the analogy a strong one. Both cases involve a target area enclosed within a fragile "container" that is at risk from a high-intensity force. Thus, both a salient surface similarity and a relatively complete structural mapping provide retrieval cues that can connect the lightbulb and radiation analogues.

Experiment 2 was performed to investigate the influence of surface and structural similarity on analogical transfer. If spontaneous retrieval depends on summation of activation from multiple features shared by the source and target, and if both surface and structural features can serve as retrieval cues, then both types of factors should influence spontaneous transfer. However, since only structural dissimilarities actually impair the analogical mapping, structural features should have a greater impact than surface features on transfer once a hint to use the analogy is provided. These hypotheses contrast with various possible alternatives, such as that different types of similarity have equivalent effects on all steps in analogical problem solving.

\footnotetext{
Method

Materials. Four story analogues to the radiation problem were written (see Appendix). These were used as source analogues, and the radiation problem was used as the target. All of the stories are variations of the lightbulb story described above. The original story is the "fragile-glass and laser" version. The other three versions were generated by varying surface and/or structural similarities to the radiation problem. To vary the surface similarity, two of the new stories employed ultrasound waves in place of lasers. ${ }^{3}$ Since laser beams are more similar to $\mathrm{X}$ rays than are ultrasound waves, the surface similarity of a laser version to the radiation problem was greater than that of an ultrasound-wave version.

In order to make the effect of the ultrasound seem plausible to subjects, the nature of the damage done to the lightbulb, and therefore the repair required, were also modified in the ultrasound versions. Instead of the filament's being broken apart, it is described as fused together and the ultrasound waves are used to jar it apart. The two types of damage and repair do not seem to differ considerably in their similarity to the destruction of a tumor, but "jarring apart" seems more simlar to "destroying" than does "fusing together." Thus, if any advantage is observed for a laser version over an ultrasound version as a prompt for the convergence solution, it can be attributed to the more salient difference in the similarity of instruments. ${ }^{4}$

The structural similarity of the stories to the radiation problem was varied by altering the constraint preventing administration of an intense force from one direction. The constraint in two of the modified versions is that none of the several machines availabie can generate a single force of sufficient intensity. The constraint of insufficient intensity is much less similar to that in the radiation problem than is the alternative constraint that a high-intensity force would break the surrounding glass. Although the same convergence solution is described in the insufficient-intensity versions, the convergence element of the solution is not essential, as it is in the fragileglass versions or in the radiation problem. Among the three basic components of the convergence solution-the application of
}

(1) multiple (2) low-intensity forces (3) from different directionsonly the use of multiple machines is a necessary component in the insufficient-intensity versions. The use of low-intensity forces in this context is a simple restatement of the given fact that only lowintensity forces are available. It is not strictly necessary to focus the forces on the filament from different directions. Another possible solution would be to focus several forces on some point outside the lightbulb so as to form a single high-intensity force. This would not be a viable solution for the fragile-glass versions, since the high-intensity force would affect the glass as well as the filament. The necessity of focusing several forces in the insufficientintensity versions arises from the physical impossibility of putting several machines in the same spatial location, and hence is implicit in the use of multiple machines. The insufficient-intensity versions thus alter a feature that influences the necessity (although not the possibility) of the stated solution. Even though the same convergence solution is provided in all four stories, the analogy with the radiation problem is structurally weaker for the insufficient-intensity than for the fragile-glass versions.

Subjects. Sixty-three University of Michigan undergraduates served in the experiment, with 16 in each of the fragile-glass conditions and the insufficient-intensity laser condition, and 15 in the insufficient-intensity ultrasound condition. Each subject was paid $\$ 3$ for participation in a 40 -min session.

Procedure. Subjects were told that the experiment consisted of several parts, involving story comprehension and problem solving. In the first part of the experiment, subjects were asked to read and summarize a story (one of the four lightbulb stories). All versions were presented with the title "The Broken Lightbulb." Subjects were allowed to refer back to the story while writing the summary. When the summarization task was complete, subjects were asked to solve several deductive-reasoning problems. This unrelated filler task was included to reduce demand characteristics that might cause subjects to relate the story task to the subsequent problems. A sheet with the radiation problem was then handed out, and subjects were required to write as many solutions as possible, without worrying about not having enough technical knowledge.

Finally, subjects filled out a questionnaire that asked (1) whether they had tried to use the lightbulb story to help solve the radiation problem; (2) what solution to the problem was suggested by the story (a prompt for additional solutions); and (3) whether they knew the problem and its solution prior to the experiment. The first and second questions served as a hint that the initial story might be useful in solving the problem. The hint would tend to equalize retrieval for all subjects, so that factors affecting only retrieval and not transfer would have little effect once the hint was provided.

\section{Results and Discussion}

The data were discarded for 1 subject in the insufficientintensity laser condition, who indicated that she had known the radiation problem and the convergence solution prior to the experiment. As in Experiment 1, all solutions clearly stating that the $\mathrm{X}$ rays should be administered from different directions were scored as convergence solutions. Table $2 \mathrm{~A}$ presents the percentage of subjects in each of the four conditions who generated the convergence solution prior to receiving a hint to consider the story. When the source was the "laser and fragile-glass" analogue, in which both instrument and constraint were similar to those of the radiation problem, $69 \%$ of the subjects spontaneously generated the convergence solution. Transfer was significantly impaired if either the surface similarity 
of the instrument or the structural constraint similarity was reduced. The ultrasound versions yielded lower solution frequencies than did the laser versions $\left[G^{2}(1)=\right.$ $4.42, p<.05]$, and the insufficient-intensity versions yielded lower solution frequencies than did the fragileglass versions $\left[G^{2}(1)=5.92, p<.05\right]$. The factors of surface and structural similarity were approximately equal in magnitude and did not interact. If dissimilarities in both were introduced (the insufficient-intensity ultrasound version), only $13 \%$ of the subjects spontaneously generated the convergence solution. These results indicate that both surface similarities and deeper structural commonalities aid in the retrieval and use of source analogues, as would be expected on the basis of a summation mechanism.

As the data in Table 2B indicate, a different transfer pattern was observed once a hint to use the story was provided. The total percentages of subjects producing convergence solutions include subjects who generated the solution either before any hint or in response to the direct question as to what solution the story suggested. Structural dissimilarity of the constraints significantly impaired total transfer ( $78 \%$ for the fragile-glass versions vs. $54 \%$ for the insufficient-intensity versions) $\left[G^{2}(1)=4.31\right.$, $p<.05]$. In contrast, surface dissimilarity of the instruments had no effect on total transfer $(68 \%$ for the laser versions vs. $64 \%$ for the ultrasound versions) $\left[G^{2}(1)<\right.$ 1]. Thus, although surface and structural similarity had comparable effects on spontaneous transfer, only the latter had a significant impact on total analogical transfer once a hint was provided.

A further analysis was performed to provide a more direct statistical test of the differing impacts of surface and structural similarity on use of the analogy. A weighted least squares analysis was used to compare the parameters of an additive linear logit model (Grizzle, Starmer, \& Koch, 1969; Wickens, in press) for the odds of solving without a hint with the parameters of a model for the odds of solving at all. The interaction of surface and structural similarity was not significant for either dependent mea-

Table 2

Percentage of Subjects Producing Convergence Solution (Experiment 2)

\begin{tabular}{|c|c|c|c|}
\hline \multirow[b]{2}{*}{$\begin{array}{l}\text { Structural Similarity } \\
\text { (Constraint) }\end{array}$} & \multicolumn{2}{|c|}{$\begin{array}{l}\text { Surface Similarity } \\
\text { (Instrument) }\end{array}$} & \multirow[b]{2}{*}{ Mean } \\
\hline & $\begin{array}{c}\text { High } \\
\text { (Laser) }\end{array}$ & $\begin{array}{c}\text { Low } \\
\text { (Ultrasound) }\end{array}$ & \\
\hline \multicolumn{4}{|c|}{ A. Prior to Hint } \\
\hline $\begin{array}{l}\text { High (Fragile } \\
\text { glass) }\end{array}$ & 69 & 38 & 54 \\
\hline $\begin{array}{l}\text { Low (Insufficient } \\
\text { intensity) }\end{array}$ & 33 & 13 & 23 \\
\hline Mean & 51 & 26 & \\
\hline \multicolumn{4}{|c|}{ B. Total (Before and After Hint) } \\
\hline $\begin{array}{l}\text { High (Fragile } \\
\text { glass) } \\
\text { Low (Insufficient }\end{array}$ & 75 & 81 & 78 \\
\hline intensity) & 60 & 47 & 54 \\
\hline Mean & 68 & 64 & \\
\hline
\end{tabular}

Table 3

Percentage of Subjects Reporting Noticing of Analogy (Experiment 2)

\begin{tabular}{lccc}
\hline & \multicolumn{2}{c}{$\begin{array}{c}\text { Surface Similarity } \\
\text { (Instrument) }\end{array}$} & \\
\cline { 2 - 3 } $\begin{array}{c}\text { Structural Similarity } \\
\text { (Constraint) }\end{array}$ & $\begin{array}{c}\text { High } \\
\text { (Laser) }\end{array}$ & $\begin{array}{c}\text { Low } \\
\text { (Ultrasound) }\end{array}$ & Mean \\
\hline $\begin{array}{l}\text { High (Fragile } \\
\text { glass) } \\
\text { Low (Insufficient } \\
\text { intensity) }\end{array}$ & 88 & 56 & 72 \\
Mean & 40 & 13 & 27 \\
\hline
\end{tabular}

sure, so only parameters for the two main effects were included in the models. The hypothesis that the parameters corresponding to the effect of surface similarity were identical for solutions obtained prior to the hint and for total solutions was rejected; the value of the Wald statistic (an approximately chi-square distributed statistic) was 4.20 with $1 d f, p<.05$. In contrast, the hypothesis of equal parameters corresponding to structural similarity could not be rejected (Wald statistic $=1.58, p>.20$ )..$^{5}$ The results of Experiment 2 therefore support the prediction that surface similarity will have a greater relative impact on retrieval of a source analogue than on application of an analogue once it is retrieved, whereas structural similarity has a comparable impact on both steps in analogy use.

Subjects' responses to the question of whether they had tried to use the prior story to help solve the radiation problem confirmed that both types of similarity affected spontaneous use of the analogy. As the data in Table 3 indicate, the percentage of subjects reporting use of the story decreased both when the instrument was dissimilar $\left[G^{2}(1)=5.30, p<.05\right]$ and when the constraint was dissimilar $\left[G^{2}(1)=13.1, p<.001\right]$.

It was claimed earlier that the insufficient-intensity constraint reduces the necessity of the use of multiple directions in the solution stated in the story. Given that aspects of a story that, subjectively, seem less important tend to be omitted when subjects write summaries (e.g., Thorndyke, 1977), it follows that subjects should be less likely to mention the use of different directions when summarizing the insufficient-intensity version than when summarizing the fragile-glass version. Accordingly, the story summaries were scored for inclusion of this aspect of the convergence solution. More subjects in the fragile-glass conditions than in the insufficient-intensity conditions mentioned the use of different directions $[66 \%$ vs. $30 \%$; $\left.G^{2}(1)=8.05, p<.005\right]$. Instrument similarity had no significant effect ( $52 \%$ for the laser versions vs. $45 \%$ for the ultrasound versions).

Although subjects in the insufficient-intensity conditions tended not to mention use of different directions in their summaries, as indicated by the above results, this does not imply that they simply ignored the stated convergence solution. The summaries were also scored for inclusion of the idea of using multiple machines, which, unlike the 
idea of using different directions, is necessary to the viability of the convergence solution in all versions. Neither similarity factor had a significant influence on the probability of mentioning multiple machines in the summary. In particular, the probability of including this aspect of the solution did not differ between the fragile-glass and the insufficient-intensity conditions ( $81 \%$ vs. $77 \%$ ). The nature of the constraint thus selectively affected the perceived importance of the use of different directions. The fact that this aspect of the convergence solution was viewed as less crucial in the insufficient-intensity stories may be related to the subsequent difficulty subjects in these conditions had in developing a convergence solution to the radiation problem, even when directed to use the prior story.

\section{GENERAL DISCUSSION}

The results of the present study begin to elucidate the conditions under which people are able to spontaneously use a known analogue stored in memory when they encounter a novel problem. Spontaneous analogical transfer is most likely to occur when the target problem shares multiple features with the source analogue. Both salient surface differences, which do not impede achievement of the critical solution, and deeper structural differences, which involve the nature of the solution constraints (Carbonell, 1983), have an impact on transfer. The results implicate a retrieval mechanism based on the summation of activation from multiple shared features that serve as retrieval cues.

Whereas both structural and surface similarities influenced the probability that an analogy would be used without an explicit hint, only structural similarity - the nature of the problem's constraint-affected subjects' ability to make use of the source analogue once its relevance was pointed out. Gentner and Landers (1985) also found evidence that superficial similarity has a greater influence on accessing than on application of analogies. Unlike surface differences, a structure-violating difference between the source and target impairs mapping and makes the analogous solution more difficult to derive, since further transformation is required to generate a solution that is viable in the target situation (Carbonell, 1983).

Clearly, the present results must be viewed as preliminary, given the narrow range of materials employed. In particular, the results should not be construed as indicating that surface features will never influence mapping once a source is selected. In Experiment 2, only a single change was introduced to create the surface-dissimilarity condition. It might well be that introduction of multiple surface dissimilarities would make it more difficult to map the components of the two analogues. In addition, surface differences will continue to impair transfer if the problem solver has difficulty in discriminating them from structural differences even after a source analogue is provided. This problem may be especially acute for inexperienced problem solvers. For example, in an experiment on analogical transfer performed with 6-year-olds, Holyoak, Junn, and Billman (1984) found that what appeared to be a minor surface dissimilarity between the source and target significantly decreased the percentage of children who were able to use the analogy even when told to use it. It may be that children who lack experience with a problem domain have greater difficulty than do adults in analyzing the causally relevant aspects of the source and target problems.

In general, surface dissimilarity may or may not influence mapping, but even when it does not, it may still impair retrieval. In contrast, structural dissimilarity is expected to affect both retrieval and mapping. The present results suggest that many of the basic mechanisms involved in analogical transfer operate in other memory and reasoning tasks. Retrieval by summation of activation can provide a general mechanism for flexibly accessing information in memory that is related to a novel input. According to the present view, the distinctive aspect of retrieval of interdomain analogies, as opposed to mundane associations, lies in the selection of appropriate features of the target to use as retrieval cues. A plausibly useful remote analogue will be one that shares multiple structural features with the representation of the target problem - the initial state, the goal state, and solution constraints (Carbonell, 1983). The appropriate features can be determined by knowledge of the target domain, coupled with skill in causal analysis. Studies of expertise in domains such as physics have revealed a shift from novices' problem representations based on surface features to experts' representations based on deeper structural features (e.g., Chi, Feltovich, \& Glaser, 1981). It follows that experts should be better able to retrieve and use analogies from other domains. Both novices and experts may use a summation mechanism to retrieve potential analogues, but the latter will be better able to focus on causally relevant features to use as retrieval cues.

The contrast between the relatively high rates of spontaneous transfer obtained in the present experiments and the much lower rates observed in previous studies is attributable to the greater similarity of the analogues used here. It may be that presence of at least one pair of highly similar problem elements, such as X rays and lasers, is, for most people, necessary to trigger retrieval. Detection of an analogy based solely on abstract structural features may be a rare event for novice problem solvers. It should be noted, however, that transfer between problems as disparate as even the most similar pair used in the present study is beyond the competence of current expert systems in artificial intelligence. Even if such a program mimicked the expertise of both a physicist and a physician, it would lack any capacity to apply its knowledge about lasers and filaments to invent a procedure for treating tumors. The mechanisms that allow humans to select useful analogies, 
such as the differentiation of structural from surface features, may provide the key to designing more flexible mechanized problem solvers.

\section{REFERENCES}

ANDERson, J. R. (1983). The architecture of cognition. Cambridge, MA: Harvard University Press.

Burstein, M. H. (1986). A model of learning by incremental analogical reasoning and debugging. In R. Michalski, J. G. Carbonell, \& T. M. Mitchell (Eds.), Machine learning: An artificial intelligence approach (Vol. 2). Los Altos, CA: Kaufmann.

CARBonell, J. G. (1983). Learning by analogy: Formulating and generalizing plans from past experience. In R. Michalski, J. G. Carbonell, \& T. M. Mitchell (Eds.), Machine learning: An artificial intelligence approach. Palo Alto, CA: Tioga Press.

Chi, M. T. H., Feltovich, P. J., \& Glaser, R. (1981). Categorization and representation of physics problems by experts and novices. Cognitive Science, 5, 121-152.

Clement, J. (1982, April). Spontaneous analogies in problem solving: The progressive construction of mental models. Paper presented at the meeting of the American Educational Research Association, New York.

DUNCKER, K. (1945). On problem solving. Psychological Monographs, 58(Whole No. 270).

GENTNER, D. (1983). Structure-mapping: A theoretical framework for analogy. Cognitive Science, 7, 155-170.

GentNER, D., \& GenTNER, D. R. (1983). Flowing waters or teeming crowds: Mental models of electricity. In D. Gentner \& A. L. Stevens (Eds.), Mental models. Hillsdale, NJ: Erlbaum.

GENTNER, D., \& LANDERS, R. (1985, November). Analogical access: A good match is hard to find. Paper presented at the annual meeting of the Psychonomic Society, Boston.

Gick, M. L., \& HolyoAk, K. J. (1980). Analogical problem solving. Cognitive Psychology, 12, 306-355.

Gick, M. L., \& HoLyonk, K. J. (1983). Schema induction and analogical transfer. Cognitive Psychology, 15, 1-38.

GiLovich, T. (1981). Seeing the past in the present: The effect of associations to familiar events on judgments and decisions. Journal of Personality \& Social Psychology, 40, 797-808.

Gleitman, H. (1981). Psychology. New York: Norton.

Grizzle, J. E., Starmer, C. F., \& Косн, G. C. (1969). Analysis of categorical data by linear models. Biometrika, 25, 489-504.

HAYes, J. R., \& SimON, H. A. (1977). Psychological differences among problem isomorphs. In N. J. Castellan, Jr., D. B. Pisoni, \& G. R. Potts (Eds.), Cognitive theory. Hillsdale, NJ: Erlbaum.

Hesse, M. B. (1966). Models and analogies in science. Notre Dame, IN: Notre Dame University Press.

Holland, J. H., Holyoak, K. J., Nisbett, R. E., \& Thagard, P. R. (1986). Induction: Processes of inference, learning, and discovery. Cambridge, MA: MIT Press.

HolyoAK, K. J. (1984). Analogical thinking and human intelligence. In R. J. Sternberg (Ed.), Advances in the psychology of human intelligence (Vol. 2). Hillsdale, NJ: Erlbaum.

HoLYOAK, K. J. (1985). The pragmatics of analogical transfer. In G. H. Bower (Ed.), The psychology of leaming and motivation (Vol. 19). New York: Academic Press.

Holyoak, K. J., JunN, E. N., \& Billman, D. O. (1984). Development of analogical problem solving skill. Child Development, 55, 2042-2055.

MCClelland, J. L., \& Rumelhart, D. E. (1981). An interactive activation model of context effects in letter perception: Part 1 . An account of basic findings. Psychological Review, 88, 375-407.

Reed, S. K., Ernst, G. W., \& Baneri, R. (1974). The role of analogy in transfer between similar problem states. Cognitive Psychology, 6, 436-450.

Ross, B. H. (1984). Remindings and their effects in learning a cognitive skill. Cognitive Psychology, 16, 371-416.
SChank, R. C. (1982). Dynamic memory. Cambridge, MA: Cambridge University Press.

SPENCER, R. M., \& WeISBERG, R. W. (1986). Is analogy sufficient to facilitate transfer during problem solving? Memory \& Cognition, 14, 442-449.

Thibadeau, R., Just, M. A., \& Carpenter, P. A. (1982). A model of the time course and content of reading. Cognitive Science, $\mathbf{6}$, 157-203.

ThORNDYKe, P. W. (1977). Cognitive structures in comprehension and memory of narrative discourse. Cognitive Psychology, 9, 77-110.

TVERSKy, A. (1977). Features of similarity. Psychological Review, 84, 327-352.

WICKENS, T. (in press). Statistical methods for frequency tables. New York: W. H. Freeman.

Winston, P. H. (1980). Learning and reasoning by analogy. Communications of the $A C M, 23,689-703$.

\section{NOTES}

1. The reader may have noticed that the summation-of-activation mechanism is itself an example of the convergence principle that can be used to solve Duncker's radiation problem (multiple low-intensity sources combine to achieve the threshold for some critical event). We will not speculate as to whether this similarity illustrates the analogical origin of scientific hypotheses, or is simply coincidental.

2. Note, however, that a structure-violating difference need not render an analogy useless. For example, even with the added constraint of having only a single $\mathrm{X}$-ray source available, a kind of convergence solution could be implemented by passing the rays through a device that defocuses them and then redirects them to a new focus at the tumor site. However, the constraint dissimilarity would make it necessary to modify the solution directly analogous to that in the source analogue.

3. The ultrasound versions were less realistic than the laser versions in that ultrasound waves will not pass through a vacuum, such as that inside a lightbulb, and hence would not, in fact, achieve the stated outcome. However, none of our subjects, most of whom were psychology majors, expressed any doubt that ultrasound waves would behave as described in the story.

4. A further experiment was performed to control for any possible effect of varying the nature of the damage and repair. This experiment used two fragile-glass versions of the lightbulb story in which both the laser and the ultrasound beam were described as being able to "jar apart" wires in the filament that had overheated and fused together. The pattern of transfer was the same as in the experiment described here. The convergence solution to the radiation problem was spontaneously generated by $87 \%$ of the 15 subjects who read the laser version, compared to $41 \%$ of the 17 subjects who read the ultrasound version $\left[G^{2}(1)=\right.$ $7.53, p<.01]$. After the subjects were given a hint to use the story, however, the two conditions did not differ significantly in solution frequencies $[87 \%$ for the laser version vs. $77 \%$ for the ultrasound version; $\left.G^{2}(1)<1\right]$.

5. We thank Tom Wickens for performing the logit analysis.

\section{APPENDIX}

Four Versions of the Lightbulb Story

\section{First part, all versions}

In a physics lab at a major university, a very expensive lightbulb which would emit precisely controlled quantities of light was being used in some experiments. Ruth was the research assistant responsible for operating the sensitive lightbulb. One morning she came into the lab and found to her dismay that the lightbulb no longer worked. She realized that she had forgotten to turn it off the previous night. As a result the lightbulb overheated, and [the filament/two wires in the filament] inside the bulb [had broken into two parts/fused together]. The surrounding glass bulb was completely sealed, so there was no way to open it. Ruth knew that the lightbulb could be repaired if a brief, high-intensity [laser beam/ultrasound wave] could be used to 
[fuse the two parts of the filament into one/jar apart the fused parts]. Furthermore, the lab had the necessary equipment to do the job.

\section{Second part, fragile-glass versions}

However, a high-intensity [laser beam/ultrasound wave] would also break the fragile glass surrounding the filament. At lower intensities the [laser/ultrasound wave] would not break the glass, but neither would it [fuse the filament/jar apart the fused parts]. So it seemed that the lightbulb could not be repaired, and a costly replacement would be required.

Ruth was about to give up when she had an idea. She placed several [lasers/ultrasound machines] in a circle around the lightbulb, and administered low-intensity [laser beams/ultrasound waves] from several directions all at once. The [beams/waves] all converged on the filament, where their combined effect was enough to [fuse it/jar apart the fused parts]. Since each spot on the surrounding glass received only a low-intensity [beam/wave] from one [laser/ultrasound machine], the glass was left intact. Ruth was greatly relieved that the lightbulb was repaired, and she then went on to successfully complete the experiment.

\section{Second part, insufficient-intensity versions}

However, the [lasers/ultrasound machines] only generated low-intensity [beams/waves] that were not strong enough to [fuse the filament/jar apart the fused parts]. She needed a much more intense [laser beam/ultrasound wave]. So it seemed that the lightbulb could not be repaired, and a costly replacement would be required.

Ruth was about to give up when she had an idea. She placed several [lasers/ultrasound machines] in a circle around the lightbulb, and administered low-intensity [laser beams/ultrasound waves] from several directions all at once. The [beams/waves] all converged on the filament, where their combined effect was enough to [fuse it/jar apart the fused parts]. Ruth was greatly relieved that the lightbulb was repaired, and she then went on to successfully complete the experiment.

Note-Differences between laser and ultrasound versions in brackets.

(Manuscript received May 15, 1986; revision accepted for publication November $17,1986$. 